\title{
Evaluation of a Microwave Phase Measurement System
}

\author{
Doyle A. Ellerbruch
}

(August 27, 1964)

\begin{abstract}
The best phase measurement sensitivity and accuracy are attained in a dual-channel balanced bridge type system. Two similar dual-channel systems are discussed in this report; these being the homodyne system and the modulated subcarrier. Both require low-frequency amplitude modulation in one of the channels; however, the homodyne system uses a balanced modulator. The difference in system theory due to the amplitude modulation difference is discussed, and the advantages of each are pointed out.

The standard phase shifter considered for these systems consists of a modified reflectometer terminated in a sliding short circuit. The requirements for a noncontacting short circuit are established, and its theoretical and experimental design data are given.

An error analysis is included, so the total error for any given system can quickly be determined. The maximum error can be derived by calculating the limits of the individual errors and adding them together. Equipments and tuning techniques that have been developed to reduce many errors to a minimum are discussed.

It is shown that either of these measurement systems are readily adaptable to automation. Samples of phase data that were taken from a few commercial components are included.
\end{abstract}

\section{Introduction}

A relative phase measurement system that will be utilized by the NBS Electronic Calibration Center for offering relative phase calibration services on most passive microwave devices has been investigated. The purpose of this report is to present the results of this investigation.

All experimental and theoretical data contained in this report were obtained using WR-90 waveguide. A discussion of the principles of operation of two similar measurement techniques is presented, the differences between these two techniques and relative advantage of each being pointed out in the section on modulation.

Development of the standard phase shifter for use with either of the measurement systems is discussed. This is followed by a discussion of the error sources which are the same for both measurement techniques.

It will be shown that the systems discussed in this report are readily adaptable to automated calibration services, which would reduce calibration time and provide continuous rather than point-by-point data. Data on various commercial microwave devices are included.

\section{System}

The basic circuit configuration used in measuring relative microwave phase shift is shown in figure 1 . Microwave power is generated by a single source and divided between the bridge arms. One arm contains a reference phase shifter, while the other contains an amplitude modulator and the device, the phase shift of which is to be measured. The microwave signal

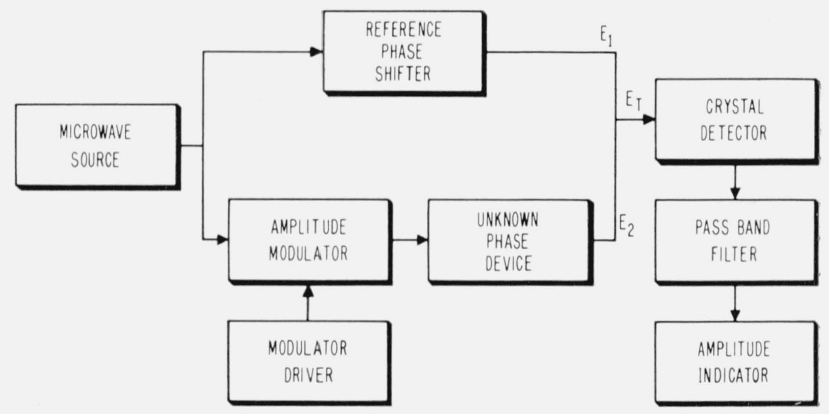

FIGURE 1. The basic phase measurement bridge circuit.

propagating through the arm containing the unknown is amplitude-modulated at a convenient low frequency.

The signals from the two arms are recombined in a crystal detector. When pure sine wave modulation is impressed, a band pass filter tuned to the fundamental modulation frequency is included as a harmonic rejector. The second harmonic arising through recombination of the carrier and modulated subcarrier is blocked by the filter, and the fundamental frequency component is allowed to proceed to the amplitude indicator.

Because the microwave signals traversing the two arms are coherent, the resultant signal present in the detector can be expressed by the use of the law of cosines. From the vector diagram shown in figure $2 \mathrm{a}$, it is seen that

$$
\left|E_{t}\right|^{2}=\left|E_{1}\right|^{2}+\left|E_{2}\right|^{2}-2\left|E_{1}\right|\left|E_{2}\right| \cos \phi,
$$

where $E_{1}$ is the amplitude of the component that reaches the detector through the standard phase 


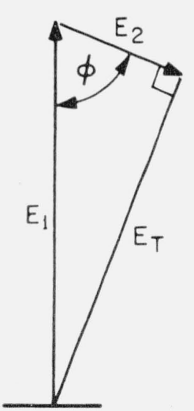

(a)

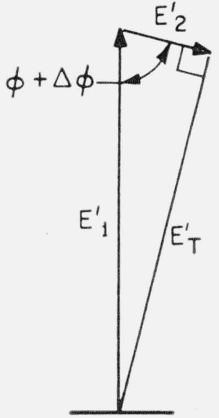

(b)
FIGURE 2. Change of angle $\varphi$ at bridge null due to a change in $E_{2} / E_{1}$.

shifter, $E_{2}$ is the amplitude of the component that reaches the detector through the modulator and phase shifter under calibration, and $E_{T}$ is the resultant amplitude incident on the detector. The power out of the square law detector is then proportional to $E_{T}^{2}$. Only those terms in (1) which contribute to the fundamental modulation frequency need be considered from here on. We have, therefore, only to consider the last two terms. This equation will be discussed in detail in the section on modulation; at this point, a general consideration of the equation is sufficient.

Considering the last two terms of (1), it can be seen that the magnitude of the modulation signal varies from zero to a maximum as the angle $\phi$ is varied. Proper bridge balance is arbitrarily assumed when the magnitude of the modulation signal is zero. This choice is made because maximum bridge sensitivity is attained at the null condition.

A change of microwave phase shift through either arm of the microwave bridge will cause a change at the detector $\phi$ and, hence, in the measured amplitude. The phase device to be calibrated (relative phase) is inserted into the system, and the reference phase shifter is adjusted to produce a null at the indicator. Then, any phase advance or retardation in the wave emerging from the unknown can be exactly counteracted by an identical change of phase in that wave through the standard. The change of phase through the unknown device is, then, exactly equal to the change of phase through the reference phase shifter.

\subsection{Modulator}

Double sideband, pure amplitude modulation, with or without carrier suppression, may be employed in this system. Either of these types of modulation is satisfactory; however, certain distinguishable characteristics must be considered when determining the type of modulation to be used.

Utilization of double sideband with unsuppressed carrier, or simple amplitude modulation, results in the modulated subcarrier phase measurement system. The theory for this system has been presented in reference [1]. ${ }^{1}$ As mentioned in the previous section,

\footnotetext{
${ }_{1}$ Figures in brackets indicate the literature references at the end of this paper.
}

only the last two terms of (1) contribute to the power into the detector at the modulation frequency. The other terms either contain no modulation frequency component or are amplitude modulated at twice the fundamental rate. Assuming square-law detection, the modulation frequency component of the voltage from the detector, $V_{0}$, is proportional to $E_{T}^{2}$; i.e.,

$$
V_{0} \propto 2 m E_{2}\left(E_{2}-E_{1} \cos \phi\right) \cos \omega_{m} t
$$

where $m$ is the modulation index at angular frequency $\omega_{m}$. According to this equation, an amplitude null occurs when $E_{1} \cos \phi=E_{2}$. It follows that the angle $\phi$ at which the null occurs is entirely dependent upon the relative magnitudes of $E_{1}$ and $E_{2}$. The angle $\phi$ at which a null occurs for various magnitude ratios has been tabulated [1] and is reproduced in this report as table 1 . It can be seen in figure $2 \mathrm{~b}$, that, if known magnitude ratio changes occur during the phase calibration, the corresponding angle change, $\Delta \phi$, must be appropriately entered in the measurement data or this data will be in error by the amount $\Delta \phi$ plus the other system errors. A procedure for averaging out any error due to ratio changes during measurement is outlined in reference [1]. When that measurement procedure is used, it is not necessary to determine the ratio change.

TABLE 1. Angular relationship at null for various relative signal levels

\begin{tabular}{c|c}
\hline $20 \log _{10}\left(E_{c} / E_{8 c}\right)$ & \multicolumn{2}{|c}{ Angle $\phi$ at null, degrees } \\
\hline & \\
\hline 0 & 0.00 \\
10 & 71.56 \\
30 & 84.26 \\
40 & 88.19 \\
50 & 89.43 \\
60 & 89.82 \\
70 & 89.94 \\
80 & 89.98 \\
90 & 89.99 \\
& 90.00 \\
\hline
\end{tabular}

Use of double sideband modulation with suppressed carrier, i.e., use of a balanced modulator in the system, results in a homodyne measurement technique discussed in reference [2]. When this type of modulation is used, only the last term in (1) contributes to the fundamental modulation frequency, whose amplitude can be given as

$$
V_{0} \propto-2 E_{1} E_{2} m \cos \omega_{m} t \cos \phi .
$$

The angle $\phi$ is the angle between the two signals shown in figure 2. From this equation, it can be seen that a null occurs when $\phi=90^{\circ}$. Further, the angle $\phi$ is not dependent upon the relative magnitudes of the signal out of each arm. Therefore, it can be seen that the advantage of an ideally balanced modulator is that the calibration data need not be corrected for changes of the amplitude ratio. This advantage is never fully enjoyed, however, because no ideally balanced modulators exist. Some residual carrier is always present from the modulator, and this residual carrier combines with the other signals as shown in figure 3 . For this condition, the tabu- 
lated data for the relative magnitude ratio presented in table 1 applies, where the magnitude $E_{s c}$ now refers to the magnitude of the residual carrier. Failure to take this angle change into account results in an error identical to that for the modulated subcarrier system, which can be corrected using the averaging process for the modulated subcarrier system [1].

A balanced modulator has been constructed [3] which is capable of suppressing the carrier to a level $45 \mathrm{~dB}$ or more below the signal incident at the modulator. If equal powers were delivered to the detector through each bridge arm, the relative phase relationship for the system would be that shown in figure $3 b$, where the angle $\phi$ is the angle in table 1 corresponding to the relative magnitude ratio of 45 $\mathrm{dB}$. If, however, the signal incident on the detector through the unknown arm remains $20 \mathrm{~dB}$ or more below that from the reference channel, the magnitude ratio is increased to $65 \mathrm{~dB}$ or more making any phase changes due to the residual carrier level change negligible as can be seen from table 1 .

\subsection{Standard}

The standard or reference phase shifter is a device which introduces the required relative phase change at the required accuracy of measurement. The standard phase shifter selected for this system is a precision waveguide section terminated by a sliding short circuit as proposed by Magid [4] and is shown in figure 4. The reasons for this choice are its broadband capability, small output signal level variation with changes of relative phase, and the good accuracy attainable when properly constructed and tuned. The symbol $\lambda g$ on figure 4 represents the precision waveguide wavelength, and $\Delta l$ is the short circuit displacement within the waveguide.

Because the position of the short circuit within the waveguide is frequently adjusted during the course of measurement, it was suggested that a noncontacting type short circuit be developed to eliminate excessive wear on the precision section. The initial requirements set up for this short circuit were a constant reflection coefficient magnitude and constant reference plane with respect to motion along the precision section. A dumbbell, or cylindrical slug type arrangement, was selected on the basis of its reported behavior [5]. Because it is extremely difficult to analyze the configuration shown in figure 5 analytically, laboratory data were collected to justify its use.

The first test was to determine the variation of reflection coefficient magnitude with frequency for fixed diameter cylinders of variable length. This was accomplished by inserting a single cylinder in a swept frequency reflectometer system. The results of this test are shown in figure 6 .

The next test series was designed to determine the separation of multiple cylinders so that the overall reflection coefficient would be optimum. Results of this test series indicated that $\lambda g / 4$ is optimum where $\lambda g$ is the wavelength in the empty waveguide.

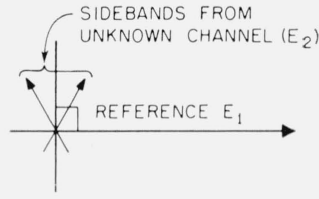

(a) CARRIER COMPLETELY SUPPRESSED.

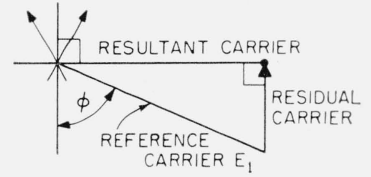

(b) RESIDUAL CARRIER PRESENT
Figure 3. Effect of residual carrier on the relative phase angle.

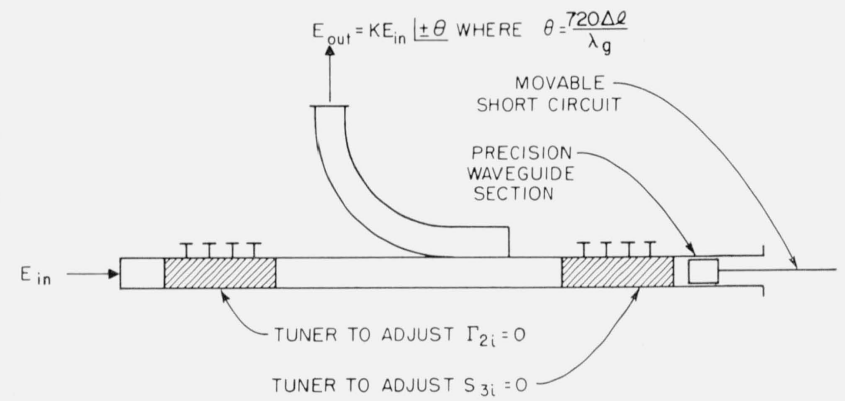

FiguRE 4. Standard phase shifter.
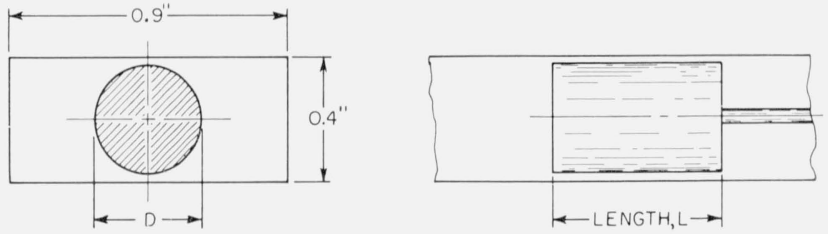

FIgURE 5. Short circuit cylinder waveguide configuration.

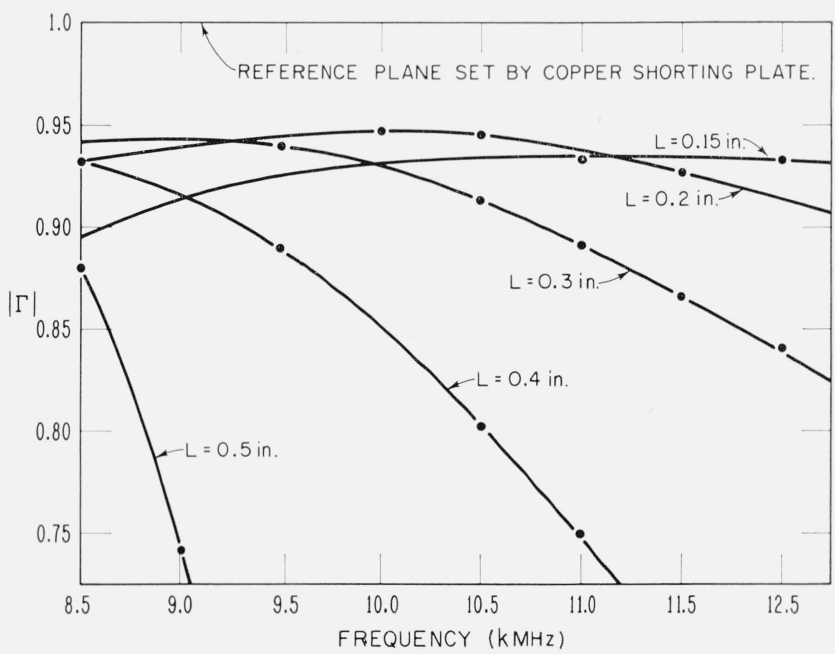

Figure 6. Reflection coefficient versus frequency for a single brass slug 0.35 in diameter. 


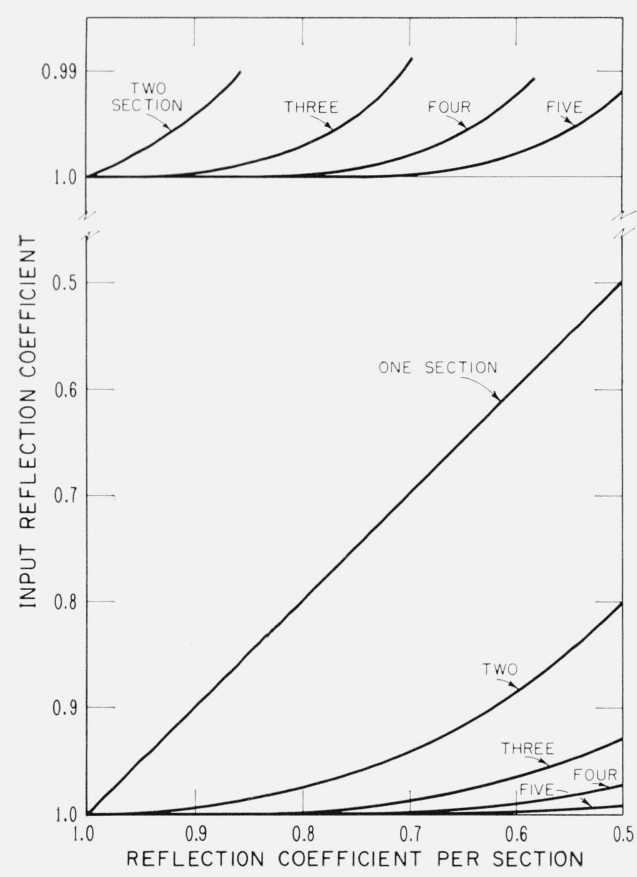

FiguRE 7. Input $|\Gamma|$ versus $|\Gamma|$ per section for multiple section short circuit.

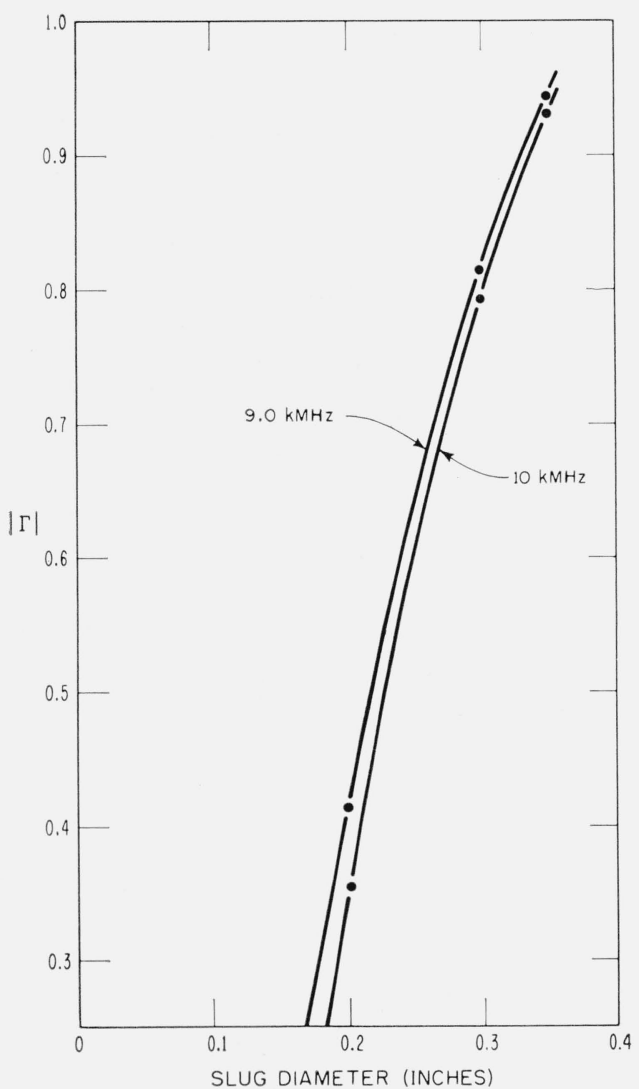

Figure 8. Reflection coefficient versus slug diameter for a single brass slug $0.30 \mathrm{in}$. long.
A theoretical curve is plotted in figure 7, showing the reflection coefficient that can be expected by cascading a number of cylinders. It was assumed that all cylinders have the same individual reflection coefficient and that the spacing between adjacent cylinders is optimum. The family of curves at the top of the figure is the same as those at the bottom; however, the input reflection coefficient scale has been changed to better show the high reflection characteristics.

The reflection coefficient of a single section as a function of cylinder diameter is shown in figure 8 , this data having been obtained experimentally. Cylinder length was constant at $0.3 \mathrm{in}$.

The final test series was conducted to determine the change of the plane of reflection, as the lateral position of the cylinder combination is changed within the precision section. The result of this test is shown in figure 9 , where the position of the cylinders within the waveguide was changed along the narrow dimension. The phase error introduced by this effect has been reduced to a negligible amount by using Teflon spacers between adjacent cylinders. The final version of the short circuit is then shown in figure 10.

Other areas of concern associated with the construction of an acceptable standard phase shifter include the dimensional tolerances of the precision section, ability to measure the relative position of the short circuit within the waveguide, and phase errors introduced by the reflectometer setup. The magnitudes of all these errors will be discussed in the error analysis section, but methods that were used to reduce these errors are discussed here.

Several types of precision waveguide constructions have been used in this system, including commercially available sections and some of special design. The best section used to the present time is shown in figure 11. It is of a four-piece construction with a cam assembly to permit adjusting of one side wall with respect to the other. This construction is easily seen in the view of a similar section in figure 12. The object of the adjustable wall is to allow for reduction of the waveguide width variation to a minimum throughout its entire length.

Different techniques of measuring the relative position of the short circuit have also been tried. The most suitable consists of a microscope-standard scale combination.

Figure 13 is a photograph of the completely assembled standard phase shifter resulting from this study.

\section{Error Analysis}

There are many sources of phase error introduced into the measurement from various parts of the overall system. These are divided into the following general categories.

\subsection{Errors From the Standard Phase Shifter}

The limits of error associated with the tuning, short circuit positioning, and precision waveguide 


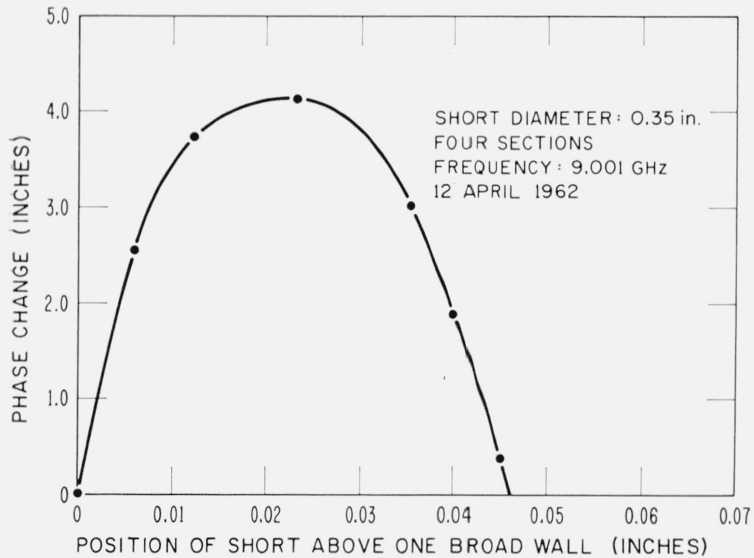

Figure 9. Change of relative phase versus position of dumbbell short in the precision guide.

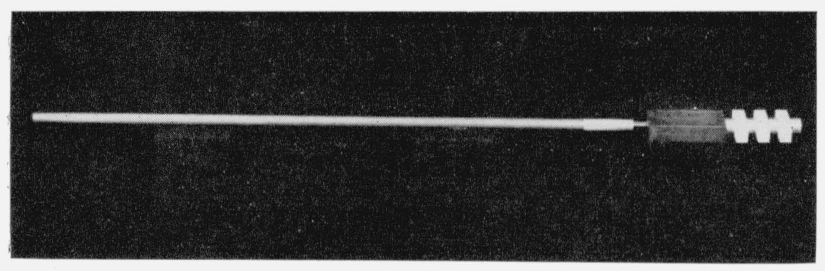

Figure 10. Complete short circuit.

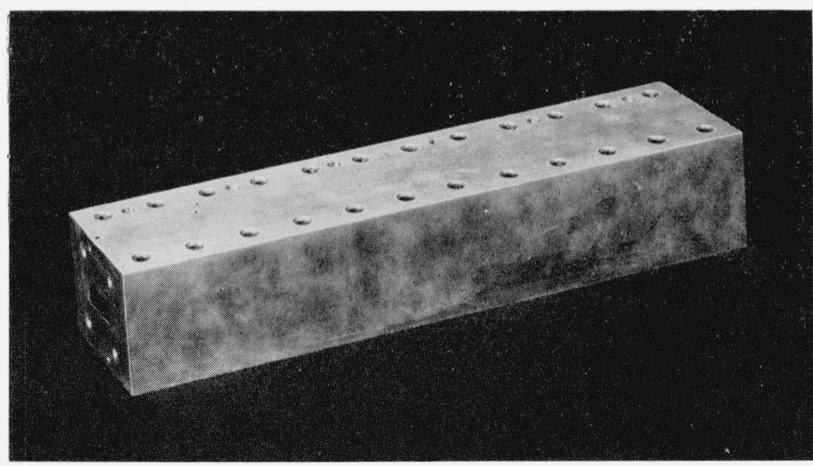

Figure 11. Adiustable waveguide section. width tolerances have been calculated and presented in graphical form [6]. The influence a reduction of the reflection coefficient to a constant value below unity has upon these errors can be determined from the mathematical results, as given by (10) and (11) in reference [6].

The error introduced in the modulated subcarrier system by a standard phase shifter, yielding attenuation changes as well as phase changes, can be determined from table 1 of this report. This error can be eliminated through use of the measurement procedure outlined in [1]. The error introduced by standard phase shifter attenuation changes in a system using a balanced modulator, where the carrier is not fully suppressed, can also be determined from table 1 . The error, in each case, is related to the magnitude ratio change of the signal emerging from the arm containing the standard phase shifter to the residual carrier, as discussed in the modulation section of this report. These errors may also be eliminated through use of the measurement procedure outlined in [1].

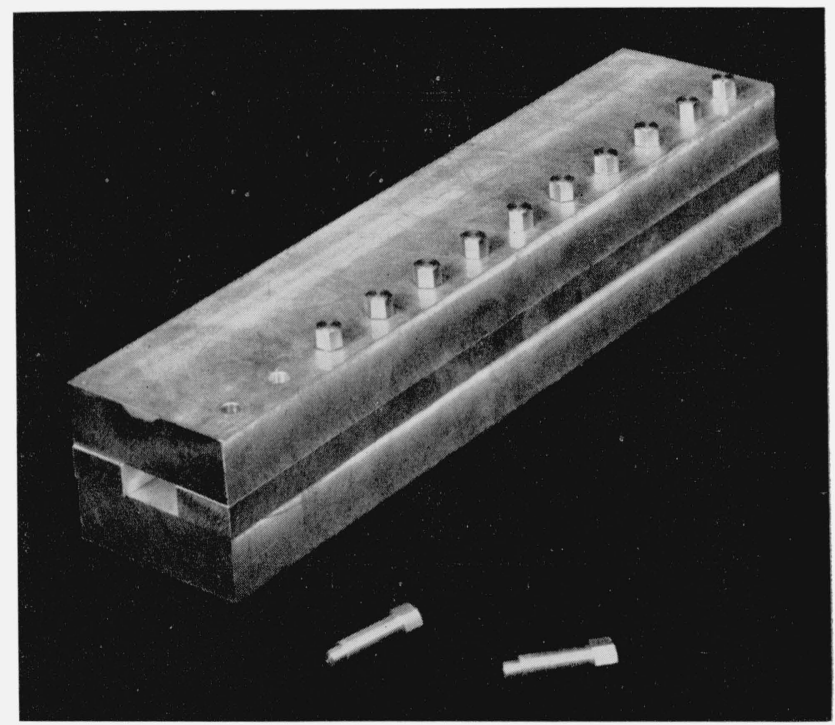

FIgure 12. Construction detail of adjustable waveguide section.

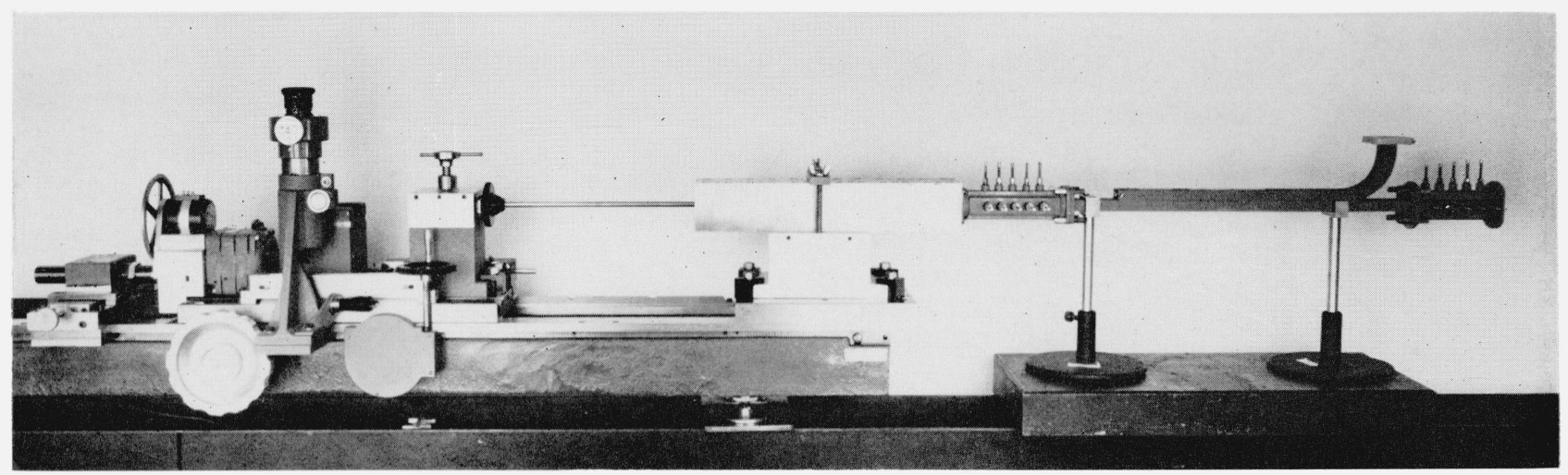

Figure 13. Standard phase shifter. 
Because the standard phase shifter relates the phase shift produced to a measured length in waveguide wavelengths, the frequency stability of the source must be considered. If the change of phase of an ideal phase shifter [6] is

$$
\psi=\frac{720 l}{\lambda g} \text { degrees, }
$$

then the phase error introduced by frequency instability is

$$
\frac{\Delta \psi}{\psi}=\frac{\frac{\partial \psi}{\partial F} \Delta F}{\psi}=\left(\frac{\lambda g}{\lambda}\right)^{2} \frac{\Delta F}{F} \frac{\text { degree error }}{\text { degree phase shift }},
$$

where $F$ is the nominal frequency of the source and $\lambda$ and $\lambda g$ are the free space and waveguide wavelengths. A graphical presentation for this error for WR-90 waveguide is shown in figure 14 .

Changes in the laboratory environment affect the dielectric constant of the air in the standard phase shifter and will, therefore, introduce a measurement error. The use of (4) and the relation

$$
\lambda g=\frac{1}{\sqrt{F^{2} \mu \epsilon-\frac{1}{(2 a)^{2}}}}
$$

gives the following error:

$$
\frac{\Delta \psi}{\psi}=\frac{\frac{\partial \psi}{\partial \epsilon} \Delta \epsilon}{\psi}=\left(\frac{\lambda g}{\lambda}\right)^{2} \frac{\Delta \epsilon}{2 \epsilon} \frac{\text { degree error }}{\text { degree phase shift }}
$$

where $\epsilon=\epsilon_{0} \epsilon_{r}$.
An approximate dielectric constant for air in the laboratory environment can be calculated through the use of an equation for the radio refractive index of air [7].

$$
N=\frac{77.6}{T}\left(P+\frac{4810 e}{T}\right)
$$

where

$N=\frac{(\epsilon-1) 10^{6}}{2}$

$\epsilon=$ the dielectric constant,

$\mathrm{P}=$ partial pressure of air in millibars,

$e=$ partial pressure of water vapor in millibars, and $T=$ absolute temperature, ${ }^{\circ} \mathrm{K}$.

Bean [7] has shown that changes in $N$, and thus $\epsilon$, due to small changes in temperature, pressure, and vapor pressure may be evaluated from the partial derivatives of (8).

$$
\begin{aligned}
\Delta N=\frac{\partial N}{\partial T} \Delta T+\frac{\partial N}{\partial e} \Delta e+\frac{\partial N}{\partial P} \Delta P \\
\quad=\frac{77.6}{T}\left(\Delta P+\frac{4810 \Delta e}{T}-\frac{P \Delta T}{T}-\frac{9620 e \Delta T}{T^{2}}\right) .
\end{aligned}
$$

Figure 15 is a graph of the limits of phase error for a variation in dielectric constant.

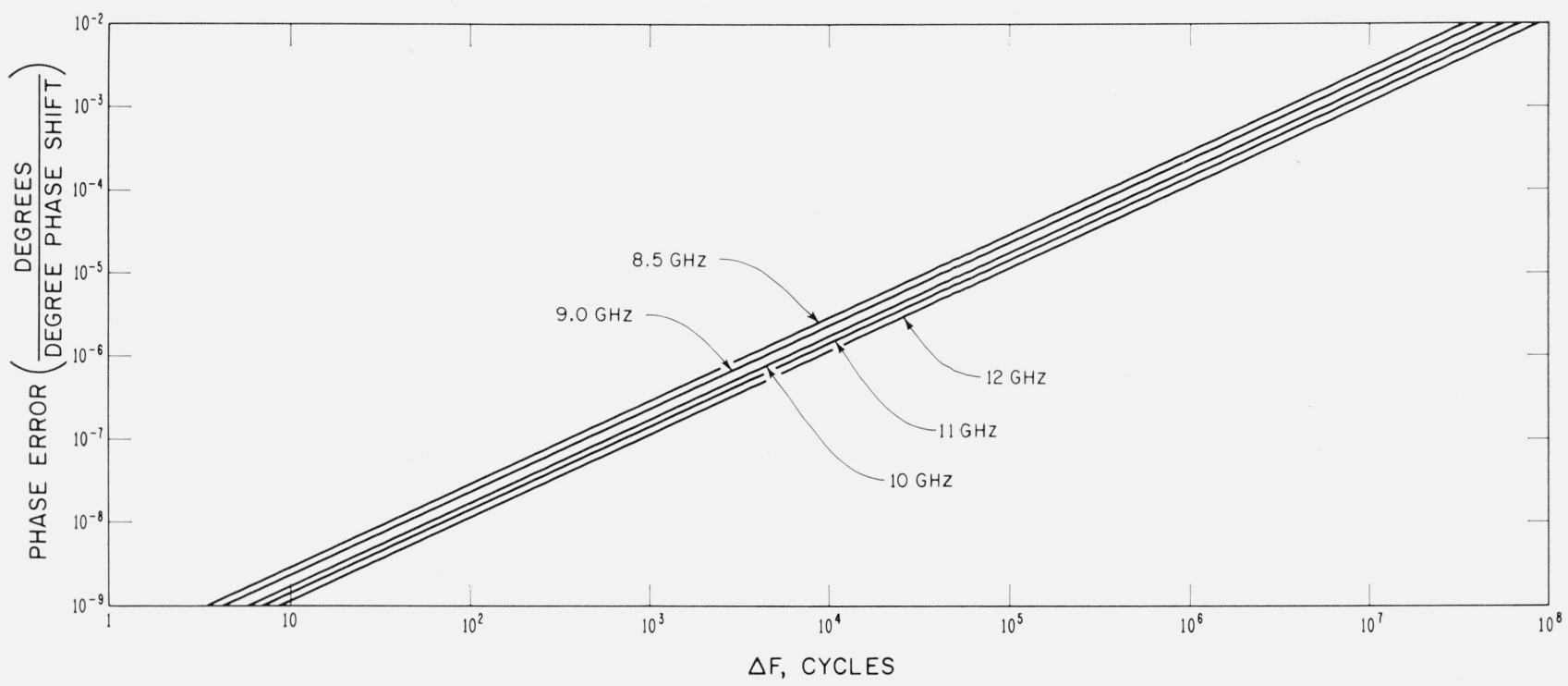

FIGURE 14. Phase error versus frequency instability for the standard phase shifter. 


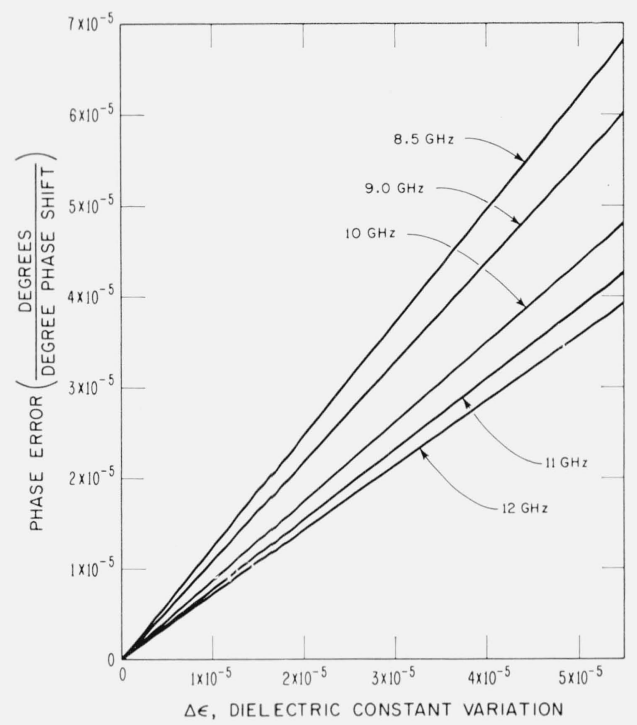

Figure 15. Phase error versus dielectric constant variation.

\subsection{Other Errors}

\section{a. Mismatch Errors}

The phase error introduced by mismatch of the unknown, when inserted into the system, has been analyzed and presented in reference [8].

\section{b. Microwave Frequency Instability Effects}

The microwave signal out of a source is propagated to the detector through two electrical paths. The physical length of these two paths will never be exactly equal in the systems discussed in this report, because the sliding short circuit will always be repositioned, thus changing the length of the reference arm.

The change of phase of the signal at the detector relative to the generator is

$$
\varphi_{1}=\frac{360 l_{1}}{\lambda g} \text { degrees through path one, }
$$

$\varphi_{2}=\frac{360 l_{2}}{\lambda g}$ degrees $=\frac{360 l_{1}}{\lambda g}+\frac{360 l^{\prime}}{\lambda g}$

degrees through path two,

$l_{1}$ being the length of path one and $\lambda g$ being the average wavelength through path one, where $l_{2}$ is the total path length through channel 2 and $l^{\prime}$ is the difference in length of arms one and two. The phase difference at the detector is then

$$
\Phi_{0}=\varphi_{2}-\varphi_{1}=\frac{360 l^{\prime}}{\lambda g} .
$$

Only the phase difference, $\Phi_{0}$, needs to be considered here, because if $\Phi_{0}=0$, then the paths are of the same length and the relative phase remains constant with changes in frequency.

The change of phase $\Phi_{0}$, with changes in frequency, can be found by determining the partial derivative of (11).

$$
\Delta \varphi_{0}=\frac{\partial \Phi_{0}}{\partial f} \Delta f=360\left(\frac{\lambda g}{\lambda^{2}}\right) l^{\prime} \frac{\Delta f}{f} \text { degrees. }
$$

A graph of the phase change described by (12) is presented in figure 16 .

\section{c. Dissimilar Changes in the Microwave Bridge Arms}

Inhomogeneous temperature variations within the laboratory, particularly those affecting the microwave bridge arms, can introduce phase differences of appreciable magnitude. An expression for the error introduced by this effect can be derived through the use of $(10)$ :

$$
\begin{aligned}
\varphi_{1} & =\frac{360 l_{1}}{\lambda g}, \\
\varphi_{2} & =\frac{360 l_{2}}{\lambda g} .
\end{aligned}
$$

Assume that the total effective bridge characteristic change occurs in arm two of the microwave bridge circuit. Then, the following relationship can be used:

$$
\varphi_{2}=\frac{360 l_{2}}{\lambda g}=\frac{360 l_{2} \sqrt{1-\left(\frac{\lambda}{2 a}\right)^{2}}}{\lambda},
$$

$\varphi_{2}$ being the total phase change throughout the length of the arm.

Inspection of (13) reveals that temperature effects upon the bridge arm can change the total phase shift through the arm and, thus, the measured relative phase shift either by changing the length $l_{2}$ or the width of the guide. It would be an extremely difficult task to determine theoretically the the amount of error introduced by each variable. The sensitivity of the system analyzed in this report to changes of this type is readily seen in figure 17 .

\section{Automation}

The system described in this report, using either of the two types of modulation discussed above, is readily adaptable for automated measurements. The objective of automation, in view of the nature of this system and the type of data that will be collected from it, is to provide for continuous monitoring of the modulation frequency component out of the detector and adjusting of the microwave phase from the reference channel to reduce the modulation frequency fundamental to zero, thus maintaining bridge balance at all times. A suitable indicator device will then display phase changes in the signal emerging from the reference phase shifter. Hence, if phase changes occur in the signal traversing 


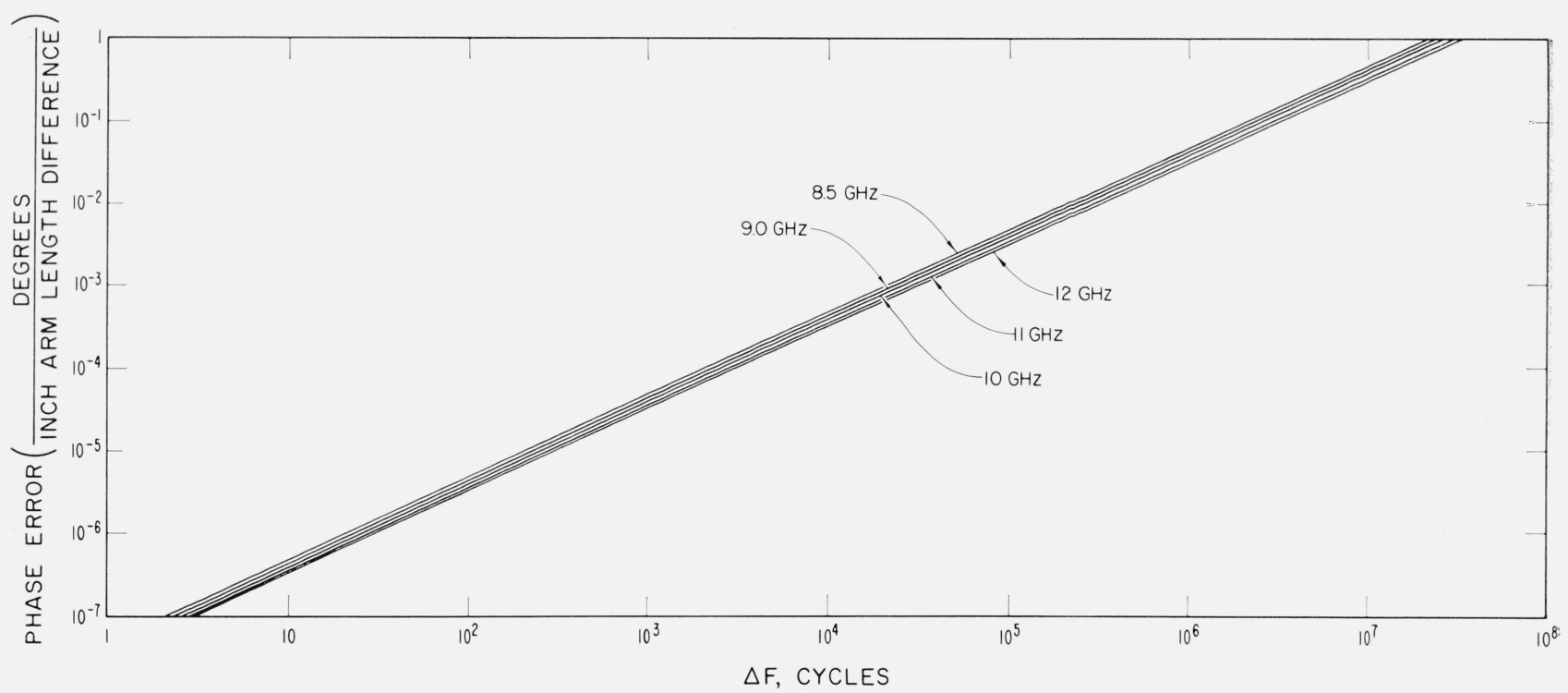

Figure 16. Phase error versus frequency instability for bridge arms of unequal length.

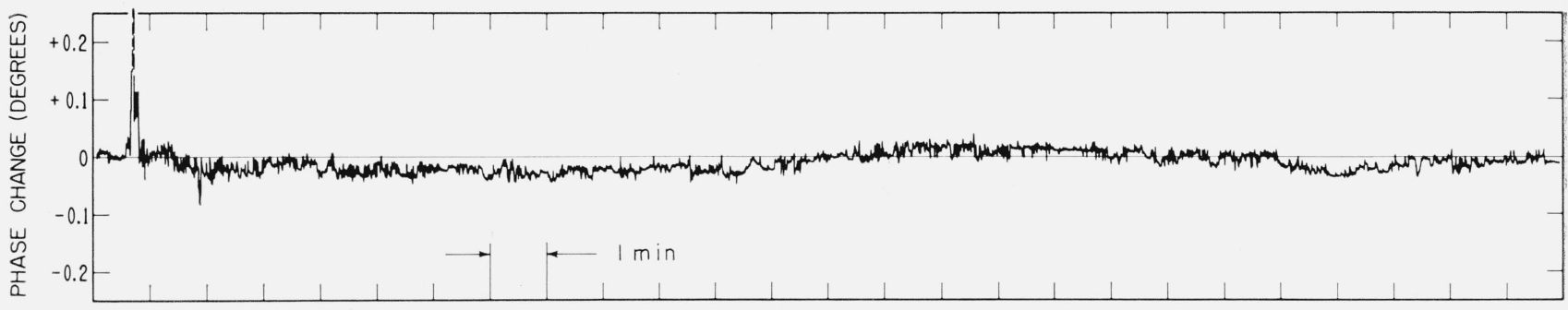

Figure 17. Phase stability of the entire system in a normal laboratory environment.

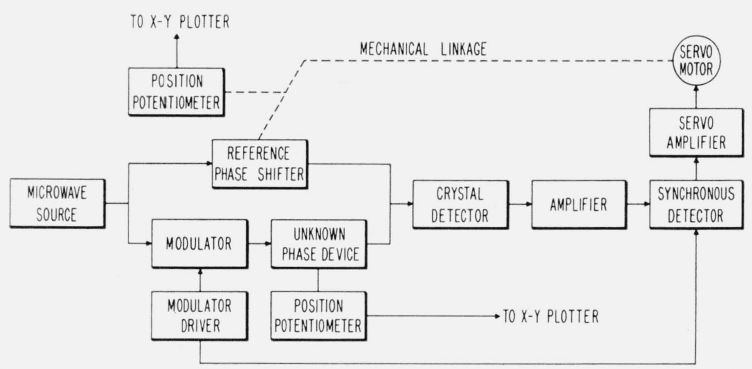

FiguRE 18. Block diagram of automation system.

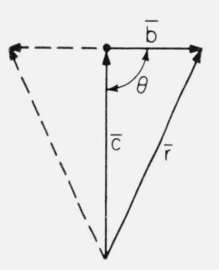

(a)

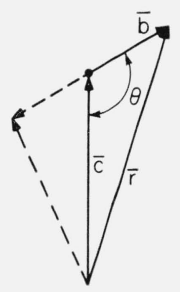

(b)

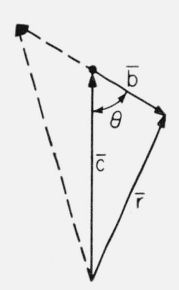

(c)

Figure 19. Derivation of servo error signal.

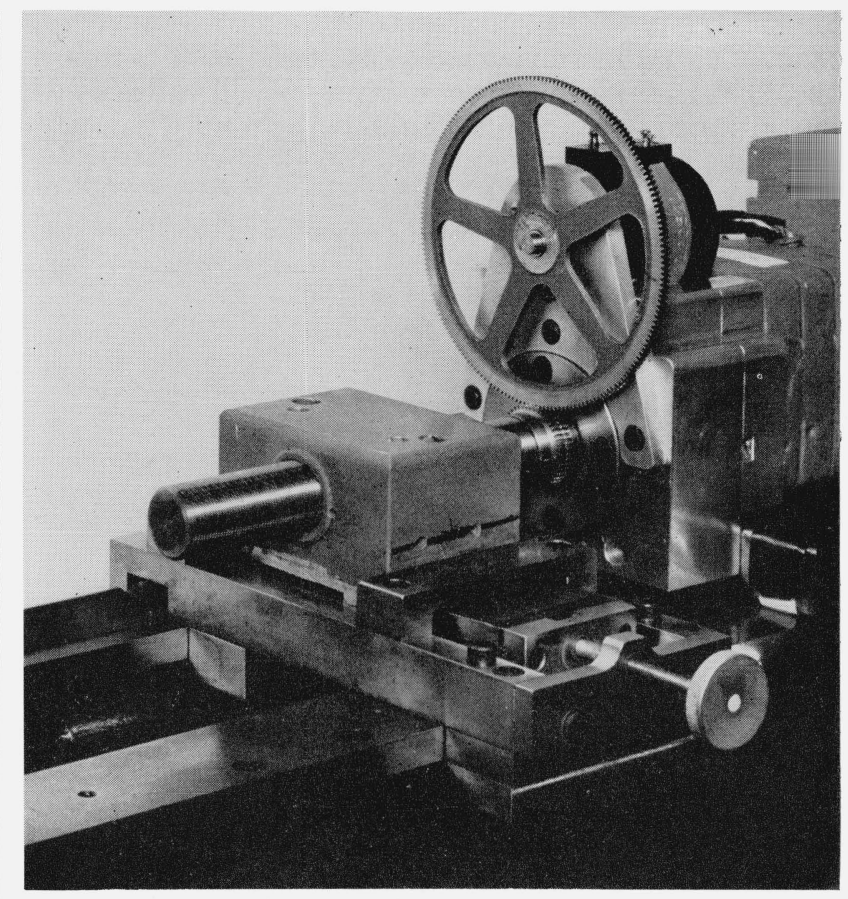

Figure 20. Position potentiometer mechanical linkage. 


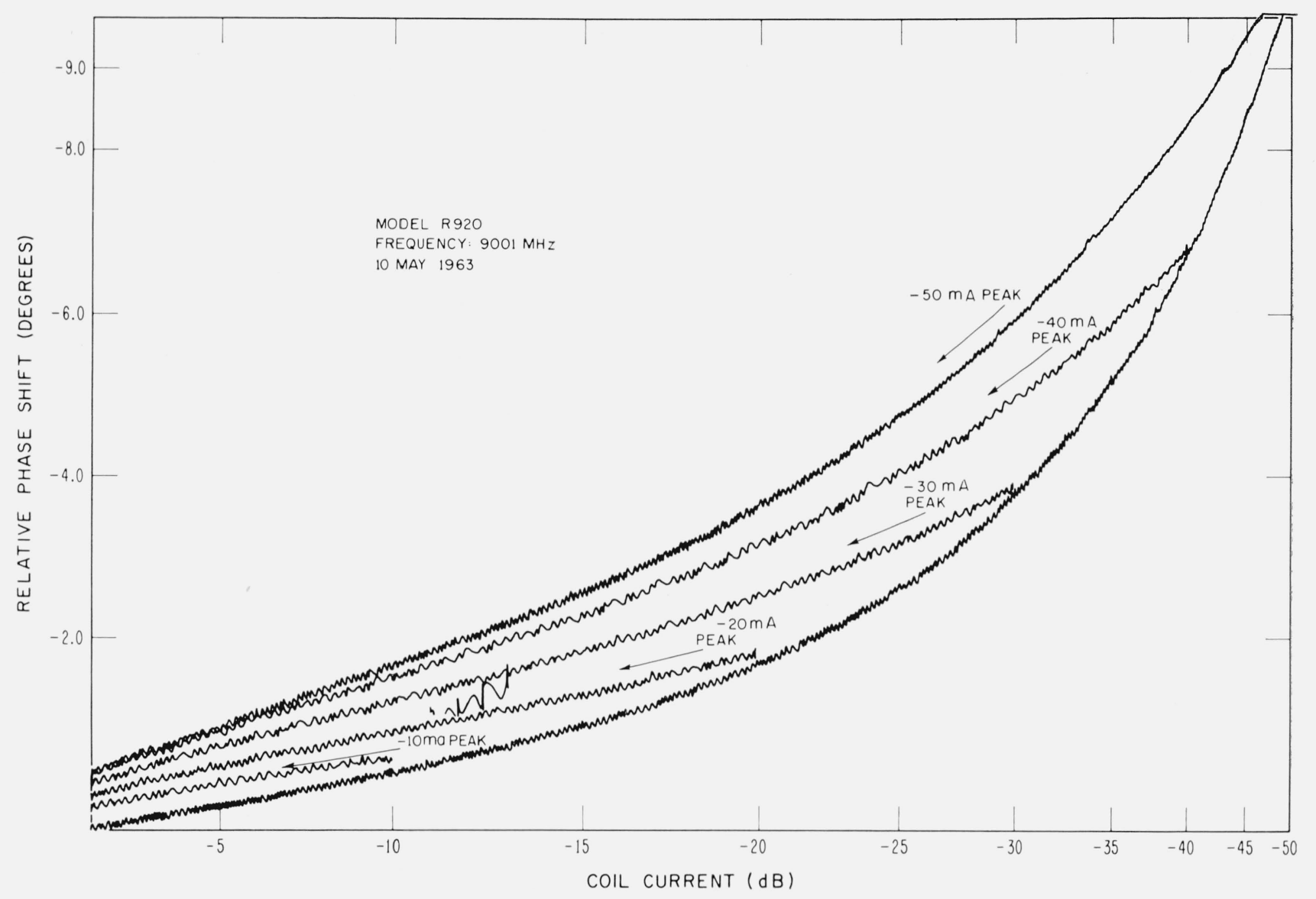

FIGURE 21a. Relative phase shift versus coil current for low attenuation side of Gyraline Model R920.

the channel containing the device under calibration, this change will be detected and automatically balanced out by a change of relative phase in the reference signal. The change of phase shift through the unknown will then exactly equal the change of phase shift through the reference phase shifter, as displayed by the indicator, to within the limits set by the measurement error.

An automatic phase tracking system capable of fulfilling this requirement has been constructed and is shown in figure 18 . The error signal used to actuate the servo system is derived in a manner which is explained vectorially in figure 19 for the balanced modulation system.

Here, $\bar{c}$ is the vector representing the unmodulated component, and $\bar{b}$ is the vector representing the modulated (suppressed carrier) component, during the positive half-cycle of the modulation. The vector $\bar{r}$ is the resultant of $\bar{c}$ and $\bar{b}$. The dotted vectors depict the situation during the negative half-cycle of the modulation. Note that in (a), where $\theta=90^{\circ}$, the resultant contains a second harmonic, but no fundamental of the modulation frequency. In (b) and (c), the fundamental is present, increasing in (b) with positively increasing $\bar{b}$, and decreasing in (c) with positively increasing $\vec{b}$. Hence, as the phase angle between the modulated and unmodulated components decreases from greater than $90^{\circ}$, through $90^{\circ}$, to less than $90^{\circ}$, the fundamental component of $\bar{r}$, decreases in magnitude to zero, reverses sign (or phase), and increases in magnitude with the new sign (or phase).

With the modulated subcarrier system a similar result is derived as the phase between carrier and subcarrier passes through the value such that the phase angle between the subcarrier and the resultant is $90^{\circ}$. In either case, the component of the resultant having the frequency of the fundamental modulation is selected by the synchronous detector and used to actuate the servo system to drive the reference phase shifter to effect a fundamental modulation frequency null in the resultant. The direction of the servo is opposite for opposite phases of the error signal, so that the reference phase shifter is always repositioned to restore the fundamental modulation frequency null illustrated by the vector relationship of figure 19a. The relative positions of both the reference phase shifter and an unknown device can be indicated automatically, in some manner. In the system used for this evaluation, linear position potentiometers are employed, their outputs being fed to an $X-Y$ plotter, which provides a direct presentation of the calibration data. Figure 20 shows the coupling 


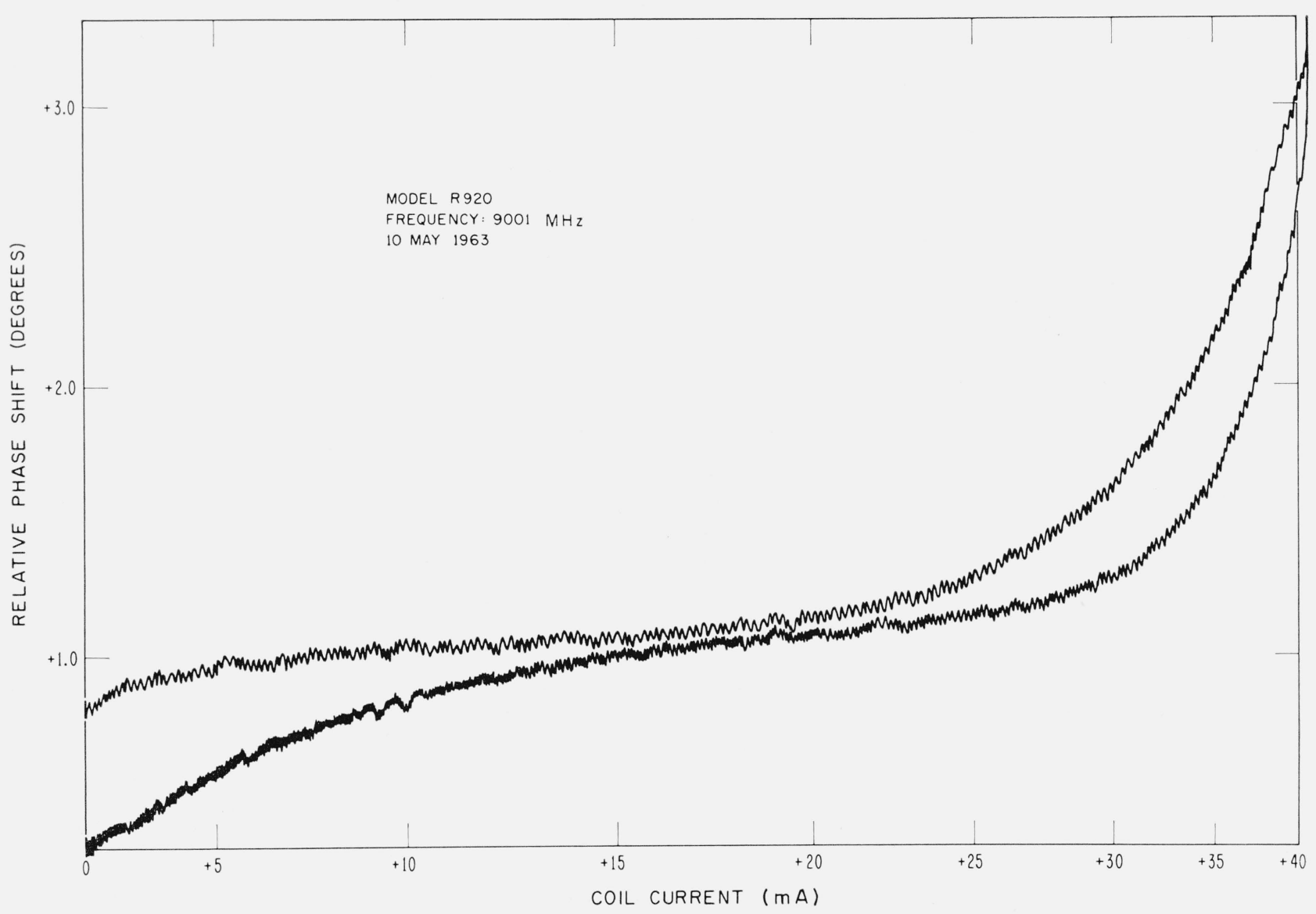

Figure 21b. Relative phase shift versus coil current for high attenuation side of Gyraline Modulator Model R920.

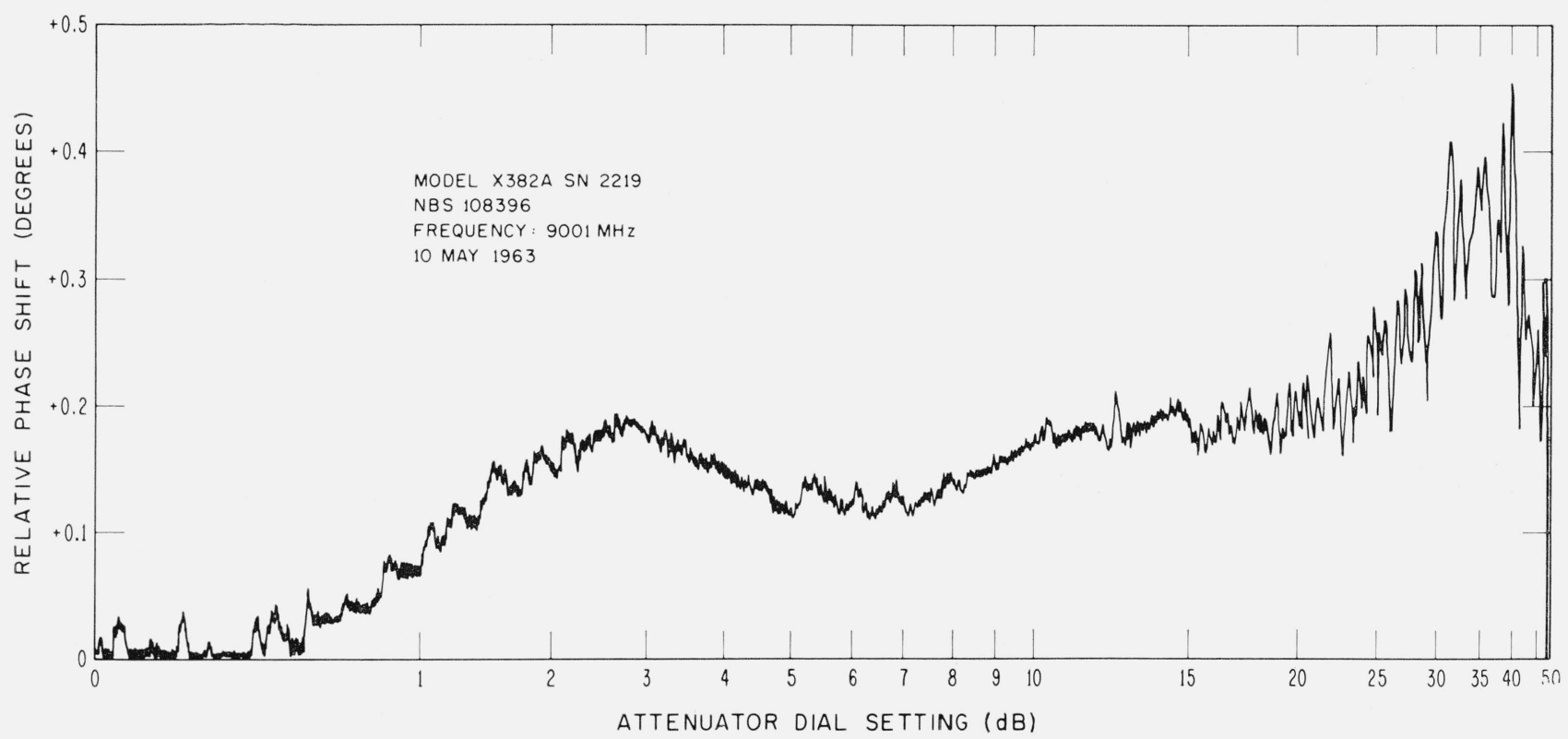

Figure 22. Relative phase shift of a Rotary Vane Attenuator. 
between the standard phase shifter and position potentiometer.

\section{System Measurement Capability}

Phase data were collected on several commercial microwave components. Figure 21 is a plot of the relative phase characteristics of a gyraline modulator. Because the attenuation characteristics vary with coil current, the servo-loop gain was set at maximum at the beginning of the run. This was done to insure maximum sensitivity of the servo system.

Because of the maximum gain, the servo system was overdriven and broke into oscillation about the null, as is evidenced by the oscillations in figure 21 . It can be seen that these oscillations resulted in a peak-to-peak microwave phase variation of approximately 0.075 deg. The actual phase shift of the device is, then, the average of this oscillation. The maximum error for this data is $0.117 \mathrm{deg}$.

Figure 22 is the phase characteristic for a commercial rotary-vane attenuator. Again the servo system was overdriven to insure maximum system sensitivity. The maximum error for this data was found to be $0.110 \mathrm{deg}$.

\section{References}

[1] G. E. Schafer (1960), A modulated subcarrier technique of measuring microwave phase shift, IRE Trans. on Instr. I-9, No. 2, 217-219.

[2] S. D. Robertson (1949), A method of measuring phase at mierowave frequencies, Bell Tech. J. 28, No. 1, 99-103.

[3] R. C. Mackey (1962), Some characteristics of microwave balanced modulators, IRE Trans. on MTT, MTT-10, No. 2, 114-117.

[4] M. Magid (1958), Precision microwave phase shift measurements, IRE Trans. on Instr. I-y, Nos. 3 and 4, 321-331.

[5] R. N. Assaly (Jan. 1961), The study of waveguide shorting plungers, Mass. Inst. Tech. Lincoln Lab. Rept. No. $315 \mathrm{G}-1,35 \mathrm{pp}$.

[6] G. E. Schafer and R. W. Beatty (Oct.-Dec. 1960), Error analysis of a standard microwave phase shifter, J. Res. NBS 64C, (Engr. and Instr.), No. 4, 261-265.

[7] B. R. Bean (1962), The radio refractive index of air, Proc. of the IRE, 50, No. 3, 260-273.

[8] G. E. Schafer (1960), Mismatch errors in microwave phase shift measurements, IRE Trans. on MTT, MTT-8, No. 6, 617-622. 